Conclusions Our data suggests the prevalence of precancerous gallbladder lesions are increasing in younger patients. Although a risk factor for cholelithiasis, BMI was not associated with disease progression. If occurring in a dysplasia-carcinoma sequence, mean age of diagnoses suggests a progression period of 20 years. Further research is required to explain the significant sex disparity and environmental risk factors for gallbladder dysplasia.

\section{PWE-37 THE LONG-TERM FOLLOW-UP OF COVID-19 RELATED LIVER INJURY}

Amelia Davies*, Fredrika Asenius, Laith Alrubaiy. Northwick Park and St Mark's Hospital, London, UK

\subsection{6/gutjnl-2021-BSG.227}

Introduction Acute COVID-19 infection is well-known to cause abnormalities in liver blood tests (LBTs). This study aimed to identify what are the long-term implications of COVID-19 on LBTs.

Methods This is a retrospective cohort study that examined the impact of COVID-19 infection on LBTs both during acute infection and for up to one year following hospital admission in 373 patients. Data analysis was done using Python using the SciPy and NumPy library. R factor was used to identify type of liver injury; hepatocellular, cholestatic or mixed. $\chi^{2}$ test and Fisher exact was used for statistical analysis with $\mathrm{p}<0.05$ being considered significant.

Results During acute infection, $57.5 \%$ of patients showed LBT abnormalities with at least one raised liver blood test (ALT, ALP and/or bilirubin). Male patients were significantly more likely to develop LBT abnormalities than were female patients $(74.5 \%$ versus $25.5 \% ; \mathrm{p}<0.001)$. The rate of LBT abnormalities was significantly correlated with severity of COVID-19 infection, such that patients requiring ITU admission were more likely to have abnormal LBTs compared to those treated on a general ward $(87 \%$ versus $51 \%$ respectively; $\mathrm{p}<0.001)$. During short term follow-up (1-5 months post discharge), LBT abnormalities persisted in $31.3 \%$ of patients. LBT abnormalities persisted for up to 12 months in $24.0 \%$ of patients. In both the acute setting and long-term follow-up, cholestatic or mixed injury types were most commonly seen (acute; $41.1 \%, \quad 41.6 \%$ respectively, long-term; 50.0\%, $44.4 \%$ respectively).

Conclusion Our data suggests that up to one in four patients have persistent LBT abnormalities up to one year following COVID-19 infection. Future research is needed to investigate what the clinical significance of this LBT abnormalities is and whether there are interventions, pharmacological or otherwise, that could reduce COVID-19 related liver injury, both in the acute setting, and longer-term.

Keywords COVID-19, coronavirus, hepatology, liver function

\section{PWE-38 THE IMPACT OF METABOLIC RISK FACTORS ON TREATED CHRONIC HEPATITIS B PATIENTS}

Almuthana Mohamed*, Patrick Kennedy, Grace Dolman. The Blizard Institute, Barts And The London School Of Medicine And Dentistry, Queen Mary University Of London, LONDON, UK

10.1136/gutjnl-2021-BSG.228
Introduction Chronic hepatitis B (CHB), as well as metabolic syndrome (MetS) and its associated risk factors, cause liver inflammation, fibrosis and cirrhosis which may subsequently lead to hepatocellular carcinoma (HCC) ${ }^{1 ; 2}$. The percentage of patients with the concomitant chronic hepatitis $\mathrm{B}$ and metabolic syndrome/non-alcoholic fatty liver disease (NAFLD) have significantly increased according to the latest reports, they stated that the prevalence of NAFLD in hepatitis B patients varies from $13.6 \%$ to $59.3 \% 3 ; 4$. However, the ramification of combined diseases on treated chronic hepatitis B patients is yet to be thoroughly explored.

Methods With the high number of chronic hepatitis B patients on treatment in our cohort; many have concomitant metabolic risk factors that may increase their risk of NAFLD, liver fibrosis, cirrhosis and subsequently hepatocellular carcinoma as well as cardiovascular risks. We aim to evaluate the extent of metabolic risk factors in our cohort of chronic hepatitis B patients and their relation to liver inflammation, fibrosis as well as renal impairment.

Our main objectives are to describe a demographic of a large cohort of patients who are on treatment for chronic hepatitis B, focusing on metabolic risk factors, to check for correlation between metabolic risk factors and liver inflammation and/or fibrosis, and to understand the effect of clinical practice on those patients.

We conducted a retrospective, descriptive, clinical-based study at Barts Health NHS Trust, London, UK. Patients who are followed for chronic hepatitis B and currently on antiviral treatment were considered for this study as part of a service evaluation. We included patients with positive $\mathrm{HBsAg}$ who are on antiviral treatment with undetected HBV DNA viral load. We excluded patients who have other comorbidities that can influence the overall results. For those who met inclusion criteria and on viral suppression, data were extracted from Barts health electronic patient records by SNOMED code with relevant demographic and clinical data including latest hepatic enzymes (ALT, AST), platelet count, Hemoglobin A1C (HBA1C), cholesterol, high density lipoprotein (HDL), transient elastography (TE) results, Biopsies and renal function including Glomerular filtration rate (eGFR) and Serum Creatinine levels. We used IBM SPSS software package v.24.0 for statistical analysis. The number of values (n), median ( $\tilde{x})$, and percentage (\%), as well as Interquartile Range (IQR), were used for describing the data. Association between metabolic risk factors and risk of liver inflammation was assessed by correlation and regression analysis techniques using both Pearson's correlation ( $r$ ) and Spearman's rank correlation along with univariate and multivariate regression analysis.

Results Eight hundred and eighty-six patients were identified as chronic hepatitis B patient on Antiviral Treatment. However, fifteen patients were excluded as they were only on Prophylactic Antiviral Treatment due to Positive Hepatitis B Core antibodies. Fifteen percent $(n=135)$ were excluded due to detectable viral replication, and fourteen percent $(n=126)$ were excluded due to other chronic conditions that may interfere with the overall results.

It was recognised in this study that nearly half of included patients were of the middle-aged group with male predominance. Given the marked gender difference in our study population, we would highlight that other gender-related results may get affected by this difference. Another pronounced result was the ethnic distribution in our study population; most of included patients were of African/Other Black/Caribbean, Asian or South Asian descents. This result can reflect the worldwide 
hepatitis B prevalence and may display the impact of immigration on the local prevalence of chronic hepatitis B as demonstrated in previous local studies $5 ; 6$.

Out of all included patients, only ten percent $(n=65)$ had a documented liver biopsy, out of which nearly half $(44.60 \%$, $\mathrm{n}=29$ ) were having fibrous portal expansion (ISHAK stage 1$2 / 6)$. And thirty percent $(n=20)$ were showing bridging fibrosis (ISHAK stage 3/6).

The levels of ALT showed marked gender discrepancy, with results ranging between 9-to-122 $\mathrm{U} / \mathrm{L}$ in males $(\tilde{\mathrm{x}}=27$, $\mathrm{IQR}=16)$, and 7 -to-102 $\mathrm{U} / \mathrm{L}$ in females $(\tilde{\mathrm{x}}=22, \mathrm{IQR}=15.25)$ ALT levels had wide variation among ethnic groups in our study. Still, the South Asian population had the most pronounced high median level of ALT. We hypothesise this may reflect NAFLD because of markedly uncontrolled T2DM combined with high BMI among this ethnic group ${ }^{7}$.

Patients who have been included in the study were assessed for correlation between metabolic risk factors represented in Body mass index (BMI), Hemoglobin A1C (HBA1C) along with Cholesterol-to-HDL ratio and changes in hepatic enzymes (ALT, AST) as an indicator for liver inflammation.
We observed that an increase in BMI, as well as HBA1C, had significantly been correlated to elevation in ALT levels. The analysis showed a moderately strong, positive and significant correlation between BMI and ALT $(r=0.380, p<0.000)$ as well as HBA1C and ALT $(r=0.323, p<0.000)$ with few outliers. However, there was no significant correlation between Cholesterol -to- HDL ratio and ALT $(r=0.0804, p=0.261)$, nor between metabolic risk factors and AST.

While reviewing the relationship between ALT and BMI at different weight ranges, results demonstrated an elevation in median levels of ALT with an increase in BMI. It also showed significantly abnormal levels among obese patients $(\tilde{\mathrm{x}}=36 \mathrm{U} / \mathrm{L}$, $\mathrm{IQR}=19$ ) compared to other groups (Overweight $\tilde{\mathrm{x}}=28 \mathrm{U} / \mathrm{L}$ $\mathrm{IQR}=15$, Normal weight $\tilde{\mathrm{x}}=23 \mathrm{U} / \mathrm{L} \mathrm{IQR}=13$ ). Out of all obese patients $(n=97), 37 \%$ had elevated ALT levels above 40 $\mathrm{U} / \mathrm{L} \quad(\mathrm{n}=36)$. In contrast, only $14 \%$ of overweight patients $(n=25)$ and $9 \%$ of normal-weight patients $(n=17)$ had elevated ALT levels above $40 \mathrm{U} / \mathrm{L}$.

When we conducted multiple regression using different independent variables; BMI and HBA1C combined has shown a powerful and significant correlation to ALT. This correlation

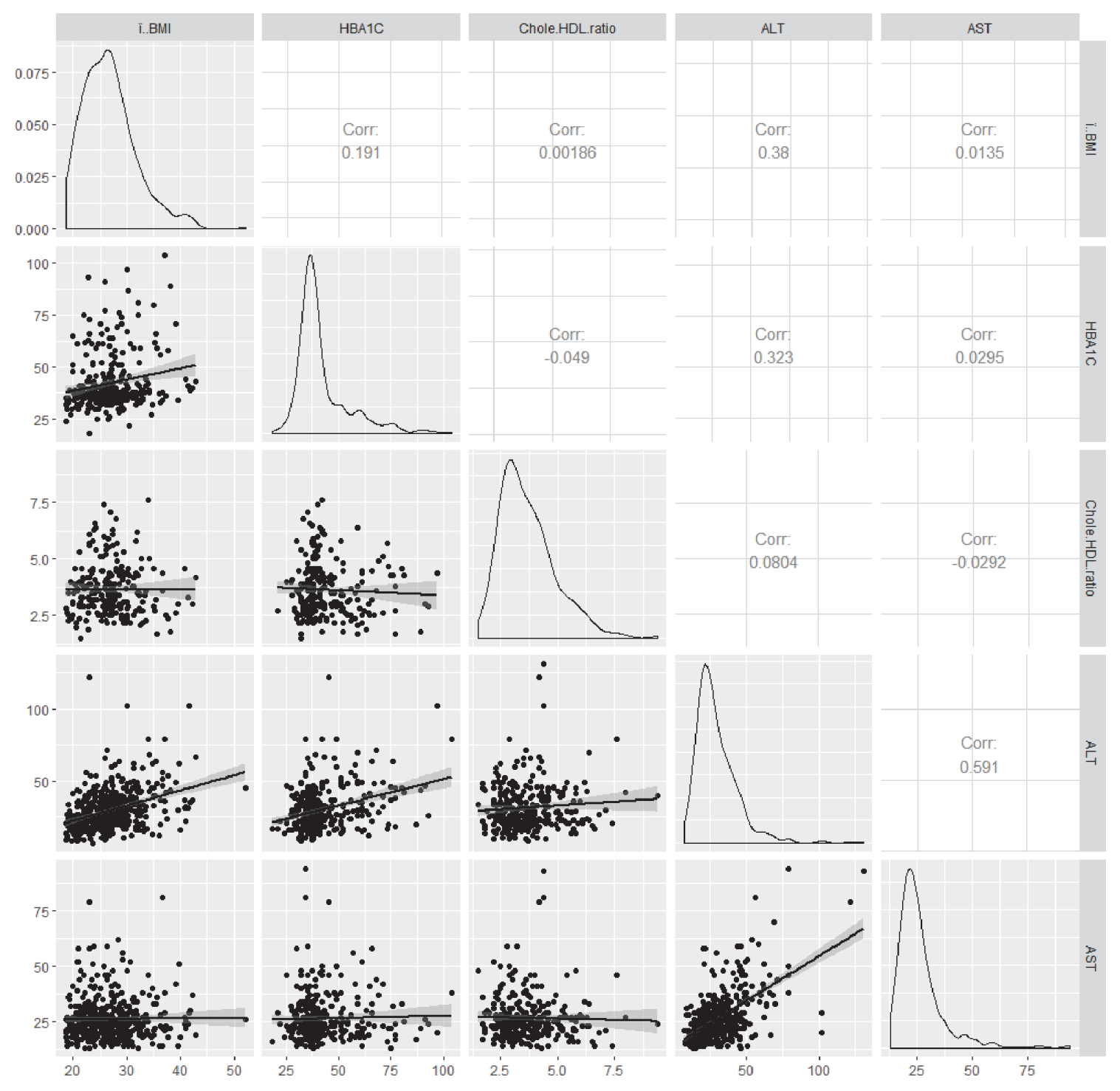

Abstract PWE-38 Figure 1 Correlation between metabolic risk factors and liver enzymes 
can be related to the effect of insulin resistance on hepatic cells and the associated fatty deposition inside the liver which lead to NAFLD as well as NASH ${ }^{8}$.

We also explored the effect of antiviral treatment, mainly Tenofovir (TDF) and Entecavir (ETV), on the renal function of CHB patients with T2DM. Results showed a significant decrease in glomerular filtration rate (eGFR) after taking antiviral medication, with median eGFR pre-treatment of $89 \mathrm{ml} /$ $\mathrm{min} / 1.73 \mathrm{~m} 2 \quad(\mathrm{IQR}=14)$ for $\mathrm{TDF}$ and $84 \mathrm{ml} / \mathrm{min} / 1.73 \mathrm{~m} 2$ $(\mathrm{IQR}=19)$ for ETV, and latest eGFR of $74 \mathrm{ml} / \mathrm{min} / 1.73 \mathrm{~m} 2$ $(\mathrm{IQR}=19)$ for $\mathrm{TDF}, 70 \mathrm{ml} / \mathrm{min} / 1.73 \mathrm{~m} 2$ for $\mathrm{ETV}(\mathrm{IQR}=20)$. Results also showed a marked increase in serum creatinine levels as well for both groups pre and post-treatment with pretreatment serum creatinine levels of $82 \mu \mathrm{mol} / \mathrm{L}(\mathrm{IQR}=22)$ for $\mathrm{TDF}$ and $86 \mu \mathrm{mol} / \mathrm{L}(\mathrm{IQR}=26)$ for $\mathrm{ETV}$, and latest serum creatinine levels of $90 \mu \mathrm{mol} / \mathrm{L}$ for TDF $(\mathrm{IQR}=21)$ and 95 $\mu \mathrm{mol} / \mathrm{L}$ for ETV $(\mathrm{IQR}=23)$. We then divided the levels of eGFR at different levels of HBA1C pre and post treatment with TDF for clinical significance, it showed a marked decrease in levels of eGFR especially in prediabetic groups, with pre-treatment median eGFR of $82 \mathrm{ml} / \mathrm{min} / 1.73 \mathrm{~m} 2$ to median eGFR of $64 \mathrm{ml} / \mathrm{min} / 1.73 \mathrm{~m} 2$ for post-treatment results.

Another perspective that should be re-considered is the role of nurse-led virtual clinics in the management of chronic hepatitis B patients. Many liver services in the UK have started to adopt the concept of virtual clinics in the follow-up of chronic hepatitis B patients for their cost-effectiveness and better compliance ${ }^{9}$. However, virtual clinics only deal with lab results and patient complaints for detection of disease flares and disease progression, but in term of screening and monitoring of metabolic risk factors such as increasing weight or elevation in blood pressure, this model can be considered ineffective.

Conclusion The effect of Metabolic syndrome and its risk factors on chronic hepatitis B patients is yet to be unravelled. The results showed a considerable number of patients with metabolic risk factors in our cohort of patients. It also presented alarming results related to the number of overweight patients and the ones with uncontrolled Type 2 Diabetes. Our study also confirmed our hypothesis of a significant and strong correlation between the degree of liver inflammation which is represented in elevated ALT levels and an Increase in both BMI and HBA1C. Even with our large study population, and the robust and strict exclusion criteria that have been implemented to strengthen this study, some limitations have been considered during the interpretation of its results. However, providing this data can give a rationale for health promotion to reduce cardiovascular risk among chronic hepatitis B patients. Dietitian, as well as Diabetes experts, should be involved in the follow-up of overweight/obese patients with elevated ALT levels to prevent disease progression and its associated complications. Given the high number of patients with uncontrolled diabetes, consideration for re-choice of antiviral drugs should be considered given the diabetic nephropathy these patients may have as well as the adverse effect they can be having from antiviral medications.

Clinicians should have a more active approach toward identifying and eliminating metabolic risk factors as well as cardiovascular risk in patients treated for chronic hepatitis B. Finally, comprehensive and longitudinal studies should be conducted for a better understanding of the extension of metabolic risk factors in our cohort and to accurately assess the associations between metabolic syndrome and chronic hepatitis $\mathrm{B}$ infection.

\section{REFERENCES}

1. EASL. EASL 2017 Clinical Practice Guidelines on the management of hepatitis $B$ virus infection. J Hepatol, v. 67, n. 2, p. 370-398, Aug 2017. ISSN 0168-8278. Disponível em: < http://dx.doi.org/10.1016/j.jhep.2017.03.021 >.

2. TERRAULT, N. A. et al. Update on prevention, diagnosis, and treatment of chronic hepatitis B: AASLD 2018 hepatitis B guidance. Hepatology, v. 67, n. 4, p. 1560 1599, 04 2018. ISSN 1527-3350. Disponível em: < https://www.ncbi.nlm.nih. gov/pubmed/29405329 >.

3. LIN, C. W. et al. Interactions of Hepatitis B Virus Infection with Nonalcoholic Fatty Liver Disease: Possible Mechanisms and Clinical Impact. Dig Dis Sci, v. 60, n. 12, p. 3513-24, Dec 2015. ISSN 0163-2116. Disponível em: < http://dx.doi.org/ 10.1007/s10620-015-3772-z >

4. MAK, L. Y. et al. Association of adipokines with hepatic steatosis and fibrosis in chronic hepatitis B patients on long-term nucleoside analogue. Liver Int, Mar 25 2019. ISSN 1478-3223. Disponível em: < http://dx.doi.org/10.1111/liv.14104 >

5. HEATHCOTE, E. J. Demography and presentation of chronic hepatitis B virus infec tion. Am J Med, v. 121, n. 12 Suppl, p. S3-11, Dec 2008. ISSN 0002-9343. Dis ponível em: < http://dx.doi.org/10.1016/j.amjmed.2008.09.024 >.

6. DIAS, A. Chronic hepatitis B infection in the immigrant communities of East London. 2014-02 2014. Disponível em: < https://qmro.qmul.ac.uk/xmlui/handle/ $123456789 / 8963>$

7. PATI, G. K.; SINGH, S. P. Nonalcoholic Fatty Liver Disease in South Asia. Euroasian J Hepatogastroenterol, v. 6, n. 2, p. 154-62, Jul-Dec 2016. ISSN 2231 5047 (Print)2231-5128 (Electronic). Disponivel em: < http://dx.doi.org/10.5005/jpjournals-10018-1189 >.

8. LONARDO, A. et al. Nonalcoholic fatty liver disease: Evolving paradigms. World J Gastroenterol, v. 23, n. 36, p. 6571-92, Sep 28 2017. ISSN 1007-9327 (Print) 2219-2840 (Electronic). Disponível em: < http://dx.doi.org/10.3748/wjg.v23. i36.6571>.

9. CORRIGALL, D. et al. PTH-086|Virtual hepatitis B clinics significantly improve cost and clinical effectiveness. 2018-06-01 2018. Disponível em: < https://gut.bmj. com/content/67/Suppl_1/A121.2 >.

\section{PWE-39 A MULTIDISCIPLINARY APPROACH TO SYMPTOMATIC UMBILICAL HERNIA IN PATIENTS WITH ESLD OPTIMISES OUTCOME}

Abdullah Malik*, Stuart Robinson, Gourab Sen, Mark Hudson, Derek Manas, Steven Masson, John Hammond. Liver Unit, Freeman Hospital, Newcastle Upon Tyne Hospitals Nhs Foundation Trust, Newcastle Upon Tyne, UK

\subsection{6/gutjnl-2021-BSG.229}

Introduction Symptomatic umbilical hernias can occur in up to $20 \%$ of patients with cirrhosis and end-stage liver disease. Management remains controversial due to a paucity of data. We aimed to determine the outcome and the factors predicting mortality following repair of symptomatic umbilical hernias in this group of patients.

Methods A retrospective review was performed for all patients with ESLD who presented to a specialist liver transplant centre with a symptomatic umbilical hernia requiring repair between 1998 and 2020. Overall survival was predicted using the Kaplan-Meier method, with censoring at transplantation. Logistic regression was used to determine predictors of 365 day mortality. Statistical significance set at $\mathrm{p}<0.05$.

Results 111 patients with ESLD underwent hernia repair (emergency $\mathrm{n}=81,73 \%$ ). Median UKELD was 51 (40-63). Mortality at $30 \mathrm{~d}$ and $365 \mathrm{~d}$ was $4.5 \%$ and $9.9 \%$, respectively. Prior to repair 28 patients $(25 \%)$ were awaiting transplantation; 19 patients (17\%) were transplanted during follow-up. Patients presenting as an emergency were more likely to have varices (13 v $0 \quad \mathrm{p}=0.018)$ and encephalopathy $(29 \quad \mathrm{v} \quad 3$ $p=0.005)$. TIPS was performed in 29 patients $(26 \%)$. Age, pre-operative bilirubin, sodium, creatinine, prothrombin time, albumin and UKELD were no different when comparing use of TIPS ( $p>0.05) .44$ patients $(40 \%)$ required ITU admission. Decompensation occurred in 44 patients $(40 \%)$, with variceal bleeding in 13 patients (12\%) and encephalopathy in 33 patients (30\%). Age $>60 \quad(\mathrm{HR} 4.5 \quad \mathrm{p}=0.026)$ and ITU 